\title{
Feh-Duf: very high-velocity low-metallicity star with peculiar chemical abundance
}

\author{
Natalia A. Drake ${ }^{1}$ and Claudio B. Pereira ${ }^{2}$ \\ ${ }^{1}$ Sobolev Astronomical Institute, St. Petersburg State University, \\ Universitetski pr. 28, St. Petersburg 198504, Russia \\ email: drake@on.br \\ ${ }^{2}$ Observatório Nacional/MCT, \\ Rua José Cristino, 77, CEP 20921-400, São Cristóvão, Rio de Janeiro, RJ, Brazil \\ email: claudio@on.br
}

\begin{abstract}
We present the results of a study of the very high velocity $\left(v_{\mathrm{rad}}=+448.0 \pm\right.$ $\left.1.0 \mathrm{~km} \mathrm{~s}^{-1}\right)$ low-metallicity $([\mathrm{Fe} / \mathrm{H}]=-1.93)$ star Feh-Duf (Fehrenbach \& Duflot 1981) showing peculiar chemical abundance. Using high-resolution spectrum, we showed that this star has enhanced carbon and heavy s-process element abundance $([\mathrm{C} / \mathrm{Fe}]=+0.58,[\mathrm{hs} / \mathrm{Fe}]=+0.88 \mathrm{dex})$ while the $[\mathrm{Y} / \mathrm{Fe}]=-0.07 \mathrm{dex})$. The carbon isotopic ratio is low $\left({ }^{12} \mathrm{C} /{ }^{13} \mathrm{C}=8\right)$. We found that oxygen abundance is reduced $([\mathrm{O} / \mathrm{Fe}]=+0.10 \mathrm{dex})$ as compared with Galactic field stars of a similar metallicity. The evolution state of this star and its possible extragalactic origin are discussed.
\end{abstract}

Keywords. stars: fundamental parameters, stars: abundances, stars: chemically peculiar, stars: kinematics, stars: Population II

\section{Introduction and Observations}

The star Feh-Duf was discovered by Fehrenbach \& Duflot (1981) during their measurements of the radial velocities of the stars in the region of the Large Magellanic Cloud. They found that this star has a very high radial velocity $\left(+440 \mathrm{~km} \mathrm{~s}^{-1}\right)$ and also that exceedingly strong $\mathrm{CH}$ absorption bands are observed in the spectrum of this star.

We present the results of the high-resolution spectroscopic study of Feh-Duf, determine its atmospheric parameters and abundance pattern, and discuss its evolutionary state and possible extragalactic origin. The high-resolution spectrum of Feh-Duf analyzed in this work was obtained with the FEROS echelle spectrograph at the $2.2 \mathrm{~m}$ telescope of ESO at la Silla, Chile, on November 20, 2008.

\section{Results and Discussion}

Using the local thermodynamic equilibrium (LTE) atmosphere models of Kurucz (1993) and the current version of the spectral analysis code MOOG Sneden (1973), we derived the following parameters for Feh-Duf: $T_{\text {eff }}=4500 \pm 120 \mathrm{~K}, \log g=0.9 \pm 0.1$, $\xi_{\mathrm{m}}=1.9 \pm 0.2$, and $[\mathrm{Fe} / \mathrm{H}]=-1.93 \pm 0.10$. The radial velocity of Feh-Duf was determined to be $v_{\mathrm{rad}}=+448.0 \pm 1.0 \mathrm{~km} \mathrm{~s}^{-1}$. Since the parallax of Feh-Duf has not been measured, we estimated its luminosity and distance using theoretical evolutionary tracks and assuming the stellar mass to be $M=0.8 M_{\odot}: M_{V}=-2.3, \log L / L_{\odot}=3.01$, and the distance $d=5.9 \mathrm{kpc}$.

The abundance analysis was performed using the LTE model-atmosphere techniques and the current version of the MOOG program. The selected lines are mainly the same used in Drake \& Pereira (2008) and Pereira \& Drake (2009). 
Our abundance analysis shows that Feh-Duf has enhanced carbon and heavy s-element abundances, $[\mathrm{hs} / \mathrm{Fe}]=+0.88$. The yttrium abundance is low, $[\mathrm{Y} / \mathrm{Fe}]=-0.07$. Comparison of the oxygen-to-iron ratio in Feh-Duf $([\mathrm{O} / \mathrm{Fe}]=+0.10)$ and in Galactic halo stars indicates that the $[\mathrm{O} / \mathrm{Fe}]$ ratio in Feh-Duf is about 0.3 dex below the corresponding value for the stars of the same metallicity in the Galaxy. Other $\alpha$-elements, such as $\mathrm{Ca}$ and $\mathrm{Ti}$, also have lower abundances $([\mathrm{Mg}+\mathrm{Ca}+\mathrm{Ti} / 3 \mathrm{Fe}]=0.23)$.

Assuming the distance to the star to be $d=5.9 \mathrm{kpc}$ we calculated Galactic spacevelocity components $(U, V, W)$ (Johnson \& Soderblom 1987). Proper motions were taken from NOMAD catalog (Zacharias et al. 2004). The obtained heliocentric space velocities are $(U, V, W)=(-116,-491,-106) \mathrm{km} \mathrm{s}^{-1}$. We transformed the $V$ component of the space velocity of Feh-Duf to the Galactic Reference Frame using $(U, V, W)_{\odot}=(9,232,7)$ (Venn et al. 2004) which results in $V_{\mathrm{GRF}}=-259 \mathrm{~km} \mathrm{~s}^{-1}$. This value of $V_{\mathrm{GRF}}$ shows that Feh-Duf has extreme retrograde motion which may be a sign that this star was accreted by the Milky Way from a dwarf satellite galaxy.

\section{Conclusions}

Our analysis of the chemical abundances and kinematic properties of Feh-Duf showed that:

- Feh-Duf is a CH star. Even though its luminosity is compatible with the early AGB phase, the binary nature of the carbon and s-element enrichment is preferable since the envelope mass of a $0.8 M_{\odot}$ star is too small for the third dredge up phenomenon to occur (Straniero et al. 2006). Feh-Duf is also a 'lead star' since its lead-to-cerium ratio is high, $[\mathrm{Pb} / \mathrm{Ce}]=+0.70$.

- The extreme retrograde motion $\left(V_{\mathrm{GRF}}=-259 \mathrm{~km} \mathrm{~s}^{-1}\right)$ may be a sign that this star was accreted by the Milky Way from a dwarf satellite galaxy. Also as pointed out by Marsakov \& Borkova (2006), a star born in a monotonically collapsing single protogalactic cloud could not be in a retrograde orbit.

- The $[\alpha / \mathrm{Fe}]$ ratio in the photosphere of Feh-Duf is lower than typical ratio for halo stars. Recently, Venn et al. (2004) carried out an analysis of the chemical abundances of the stars in the Galaxy and in the Milky Way dwarf spheroidal (dSph) satellite galaxies. They confirmed that the $[\alpha / \mathrm{Fe}]$ ratios of most stars in the dSph galaxies are lower than Galactic stars of similar metallicity.

The large retrograde velocity and the unusual abundance ratios suggest that Feh-Duf may have originated within a dSph galaxy that experienced a different nucleosynthetic chemical evolution history and has been captured by the Milky Way.

\section{References}

Anders, E. \& Grevesse, N. 1989, Geochim. et Cosmochim. Acta, 53, 197

Drake, N. A. \& Pereira, C. B., 2008, AJ, 135, 1070.

Fehrenbach, Ch. \& Duflot, M. 1981, A\& A, 101, 226

Johnson, D. R. H. \& Soderblom, D.R. 1987, AJ, 93, 864

Kurucz, R. L. 1993, CD-ROM 13, Atlas9 Stellar Atmosphere Programs and $2 \mathrm{~km} / \mathrm{s}$ Grid (Cambridge: Smithsonian Astrophys. Obs)

Marsakov, V. A. \& Borkova, T. V. 2006, Astron. Letters, 32, 545

Pereira, C. B. \& Drake, N. A. 2009, A\& A, 496, 791

Sneden, C. 1973, Ph.D. Thesis, Univ. of Texas

Straniero, O., Gallino, R., \& Cristallo, S. 2006 Nuclear Physics A, 777, 311

Venn, K. A., Irwin, M., Shetrone, M. D., Tout, C. A., Hill, V., \& Tolstoy, E. 2004, AJ, 128, 1177

Zacharias, N., Monet, D. G., Levine, S. E., et al. 2004, AAS, 205, 4815 